\title{
GOx-assisted synthesis of pillar[5]arene based supramolecular polymeric nanoparticles for targeted/synergistic chemo-chemodynamic cancer therapy
}

Jin Wang ${ }^{\dagger}$, Di Wang ${ }^{\dagger}$, Moupan Cen, Danni Jing, Jiali Bei, Youyou Huang, Jiannan Zhang, Bing Lu, Yang Wang ${ }^{*}$ and Yong Yao* (i)

\begin{abstract}
Background: Cancer is the most serious world's health problems on the global level and various strategies have been developed for cancer therapy. Pillar[5]arene-based supramolecular therapeutic nano-platform (SP/GOx NPs) was constructed successfully via orthogonal dynamic covalent bonds and intermolecular $\mathrm{H}$-bonds with the assistance of glucose oxidase (GOx) and exhibited efficient targeted/synergistic chemo-chemodynamic cancer therapy.

Methods: The morphology of SP/GOx NPs was characterized by DLS, TEM, SEM and EDS mapping. The cancer therapy efficinecy was investigated both in vivo and in vitro.

Results: SP/GOx NPs can load drug molecules (Dox) and modify target molecule (FA-Py) on its surface conveniently. When the resultant FA-Py/SP/GOx/Dox NPs enters blood circulation, FA-Py will target it to cancer cells efficiently, where $\mathrm{GOx}$ can catalyst the overexpressed glucose to generate $\mathrm{H}_{2} \mathrm{O}_{2}$. Subsequently, the generated $\mathrm{H}_{2} \mathrm{O}_{2}$ in cancer cells catalyzed by ferrocene unit to form $\bullet \mathrm{OH}$, which can kill cancer cells. Furthermore, the loaded Dox molecules released under acid microenvironment, which can further achieve chemo-therapy.
\end{abstract}

Conclusion: All the experiments showed that the excellent antitumor performance of FA-Py/SP/GOx/Dox NPs, which provided an new method for pillar[5]arene-based supramolecular polymer for biomedical applications.

Keywords: Pillar[5]arene, Supramolecular polymers, Synergistic therapy, Host-guest interactions

*Correspondence: ywang85@ntu.edu.cn; yaoyong1986@ntu.edu.cn

${ }^{\dagger}$ Jin Wang and Di Wang contribution equally to this work

School of Chemistry and Chemical Engineering, Nantong University,

Nantong, Jiangsu 22 6019, People's Republic of China

(c) The Author(s) 2022. Open Access This article is licensed under a Creative Commons Attribution 4.0 International License, which permits use, sharing, adaptation, distribution and reproduction in any medium or format, as long as you give appropriate credit to the original author(s) and the source, provide a link to the Creative Commons licence, and indicate if changes were made. The images or other third party material in this article are included in the article's Creative Commons licence, unless indicated otherwise in a credit line to the material. If material is not included in the article's Creative Commons licence and your intended use is not permitted by statutory regulation or exceeds the permitted use, you will need to obtain permission directly from the copyright holder. To view a copy of this licence, visit http://creativecommons.org/licenses/by/4.0/. The Creative Commons Public Domain Dedication waiver (http://creativeco mmons.org/publicdomain/zero/1.0/) applies to the data made available in this article, unless otherwise stated in a credit line to the data. 


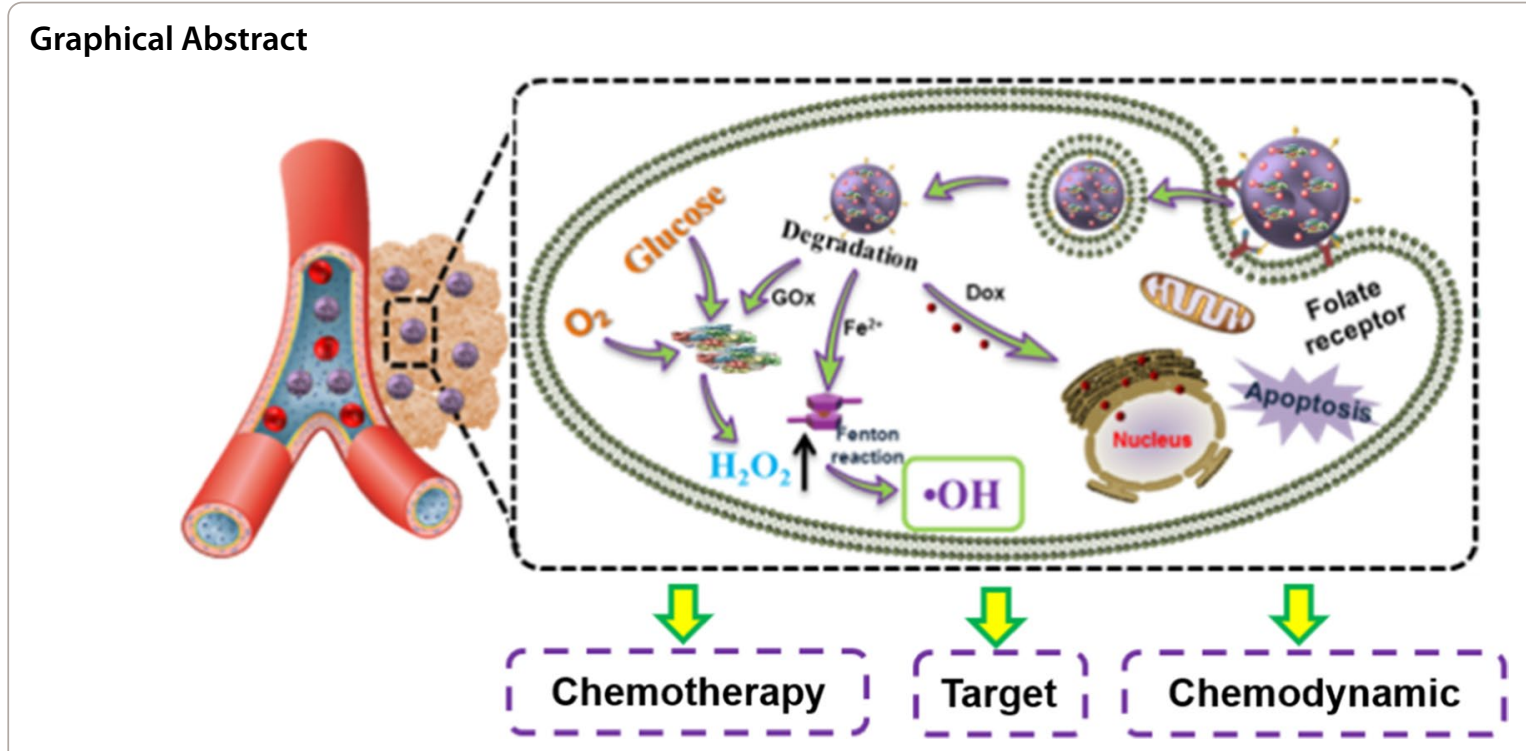

\section{Background}

Cancer is the most serious world's health problems on the global level and various strategies have been developed for cancer therapy [1]. During the past decade, supramolecular chemotherapy has obtained considerable interests and became an effective strategy for cancer treatment due to the dynamic and reversible nature of supramolecular chemistry endew the obtained materials with outstanding stimuli-responsibilities and infinite possibilities $[2,3]$ Compared with conventional chemotherapy, supramolecular chemotherapy possesses several advantages. Firstly, after forming host-guest complex or supramolecular assemblies, the solubility and stability of the anticancer drugs can be improved significantly [4]. Secondly, different functional groups, such as target units, fluorescent groups and pro-drugs, can be easily modified into systems by dynamic and reversible supramolecular interactions [5]. Thirdly, due to the differences in environments between the normal and tumor tissues, the release of the loaded drug-molecules can be precisely achieved [6]. Macrocylics, such as cyclodextrins, calixarenes and pillararenes, are the ideal platforms for construction of chemotherapeutic platforms through supramolecular interactions due to they contained sizecontrollable cavities where guests can be penetrated [7, 8].

Pillar[5]arenes, a new class of oligomeric macrocycles, were constructed from 1,4-dimethoxybenzene or its derivatives connected by $-\mathrm{CH}_{2}-$ on their $p$-positions [9-11]. Compared with other classical macrocycles, such as cyclodextrins [12-15] and cucurbiturils [16, 17], the modification of pillar[5]arenes is more convenient. On the other hand, compared with crown ethers [18-20] and calixarenes [21-23]. pillar[5] arenes possess a more rigid structures and rich host-guest properties [24-37]. So, in the past 13 years, the investigation of pillar[5]arenes is becoming a research hotspot and attracted considerable interests [38-51], especially for supramolecular polymeric materials based on pillar[5]arene [52-58]. Until now, various stimuli-responsive (including light, heat, $\mathrm{pH}$ and so on) pillar[5]arene-based supramolecular polymers have been prepared successfully and their physicochemical properties and morphologies transformation also have been studied [59-63]. For example, Prof. Wang and $c o$-workers constructed a novel pillar[5]arene-based supramolecular cross-linked polymeric pseudo[2] rotaxanes, which showed heat, $\mathrm{pH}$ and concentration stimuliresponsiveness $[64,65]$. Recently, our group constructed a Pd nanoparticles hybrid pillar[5]arene based supramolecular, and applied it in catalytic reduction of toxic nitroaromatics and catalytic Suzuki-Miyaura reaction in water [66]. Although numerous pillar[5]arene-based supramolecular polymers and their applications have been reported, the application of them in bio-medical areas, especially in targeted and synergistic cancer therapy has rarely investigated due to their poor stability [67].

Herein, a new pillar[5] arene-based supramolecular therapeutic nano-platform (SP/GOx NPs) was constructed from amino group modified pillar[5]arene (AP5, Scheme 1) and ferrocene dicarbaldehyde (FeE, Scheme 1) via orthogonal dynamic covalent bonds and intermolecular $\mathrm{H}$-bonds with the assistance of glucose oxidase (GOx). The obtained SP/ GOx NPs possess several advantages. Firstly, it can be used as drug carrier to load Dox efficiently. Secondly, the cavity 

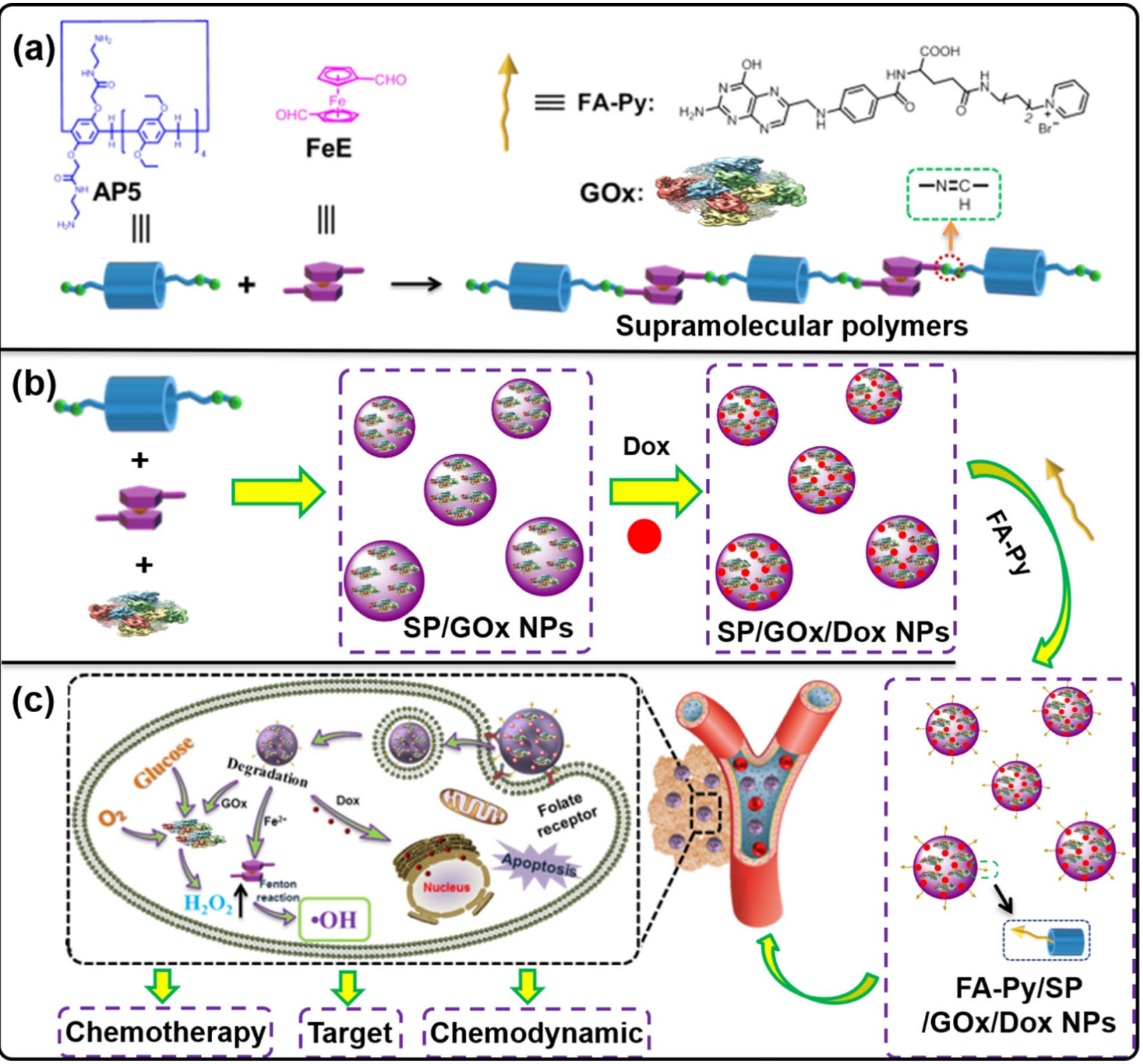

Scheme 1. a Chemical structures of amino group modified pillar[5]arene (AP5), ferrocene dicarbaldehyde (FeE), and target molecule FA-Py. b The illustration of preparation and modification of pillar[5]arene-based supramolecular therapeutic nano-platform. $\mathbf{c}$ The targeted synergistic chemo-chemodynamic cancer therapy of the multifunctional FA-Py/SP/GOx/Dox NPs

of pillar[5]arene endow SP/GOx NPs with excellent hostguest properties, so target molecules (FA-Py) can be introduced on its surface easily. In this case, when the resultant pillar[5]arene-based FA-Py/SP/GOx/Dox NPs enters blood circulation, FA-Py will target it to cancer cells efficiently. Subsequently, GOx catalyst the over expression of glucose in cancer cells to form $\mathrm{H}_{2} \mathrm{O}_{2}$ continuously, then bridged ferrocene unit will catalysis the $\mathrm{H}_{2} \mathrm{O}_{2}$ to generate $\cdot \mathrm{OH}$, to achieve chemodynamic therapy. Furthermore, the loaded Dox molecules will be released under low $\mathrm{pH}$ micro-environment in tumor issues, which will achieve synergistic chemotherapy. In vitro experiments showed that the excellent antitumor performance of FA-Py/SP/GOx/Dox NPs, which provided an new method for pillar[5]arene-based supramolecular polymer for biomedical applications.

\section{Materials and methods}

Synthesis of AP5 (See Additional file 1)

Construction of FA-Py/SP/GOx/Dox NPs

For the synthesis of SP/GOx NPs, AP5 $(9.8 \mathrm{mg}$, $0.01 \mathrm{mmol}), \mathrm{FeE}(5.0 \mathrm{mg}, 0.020 \mathrm{mmol})$, and GOx (10 mg) were dissolved in the methanol/acetone mixture and then 
stirred at $60{ }^{\circ} \mathrm{C}$ overnight under $\mathrm{N}_{2}$. The SP/GOx NPs was obtained after washing with DMF, and $\mathrm{H}_{2} \mathrm{O}$ for several times via centrifugation $(8,000 \mathrm{rpm} \times 8 \mathrm{~min})$. Then SP/GOx NPs was added to the aqueous solution of Dox and stirred overnight to obtain Dox loaded materials (SP/ GOx/Dox NPs). At last, SP/GOx/Dox NPs was added to the solution of FA-Py overnight to get resultant materials FA-Py/SP/GOx/Dox NPs.

\section{Host-guest interaction}

To determine the host-guest interaction for the complexation between AP5 and target molecule FA-Py, NMR titrations were done with solutions which had a concentration of $4.0 \mathrm{mM}$ of $\mathrm{G}$ upon different concentration of AP5.

\section{Dox loading and release}

To prepare Dox-loaded SP/GOx NPs, SP/GOx NPs was added into the solution of Dox and then stirred $24 \mathrm{~h}$. Then, the SP/GOx/Dox NPs was separated by washing with DMF and water 5 times and further centrifuging. Due to Dox contains a characteristic absorption at $490 \mathrm{~nm}$ under UV-vis spectrometer, the Dox loading capacity can be calculated by UV-vis spectrum: Dox loading capacity $=\left(\mathrm{W}_{\text {initial Dox }}-\mathrm{W}_{\text {Dox in supernatant }}\right) / \mathrm{W}_{\mathrm{SP} /}$ GOx NPs $\left(\mathrm{mg} \mathrm{mg}^{-1}\right)$.

The Dox release was carried out by UV-vis spectrum in different $\mathrm{pH}$ values $(7.0,6.0,4.8$ and 3.5) of PBS solution.

\section{In vivo antitumor efficacy}

When the tumor volume grew to about $100 \mathrm{~mm}^{3}$, all the mice were randomly divided into 7 groups $(n=5)$ and intravenously injected with Control, SP NPs, SP/GOx NPs, FA-Py/SP/GOx NPs, SP/Dox NPs, FA-SP/Dox NPs, and FA-Py/SP/GOx/Dox NPs for every three days. The tumor volume and body weight of the mice were monitored. The normal organs and tumor tissues were collected and investigated by H\&E staining at 21th day.

\section{Results}

\section{Preparation and characterization of SP/GOx NPs}

Amino-amide group modified pillar[5]arene (AP5) was designed and prepared in two steps. As shown in Scheme S1, copillar[5]arene 3 was first prepared from condensation of monomer 1 and $2(\mathbf{1}: \mathbf{2}=1: 4)$ with $\mathrm{BF}_{3} \cdot \mathrm{Et}_{2} \mathrm{O}$ as the catalyst. Then AP5 was obtained by refluxing a solution of 3 and excess 1,2-ethanediamine in ethanol. With AP5 in hands, supramolecular polymeric nanoparticles with (SP/GOx NPs) or without (SP NPs) GOx were constructed in DMSO at $80{ }^{\circ} \mathrm{C}$ overnight by employing AP5 and ferrocene dicarbaldehyde (FeE) as building blocks. In order to investigate the change of AP5 and FeE during the construction of polymers, ${ }^{1} \mathrm{H}$ NMR of the chemical species against time was performed. As shown in Additional file 1: Fig. S6, the proton of the aldehyde groups on FeE became smaller and smaller as the reaction proceeds, and disappeared after $40 \mathrm{~min}$ (Additional file 1: Fig. S6e), indicating that the carbazone reaction was completed.

The morphology of SP/GOx NPs was then characterized by dynamic light scattering (DLS), transmission electron microscopy (TEM) and scanning electron microscopy (SEM) images. As shown in Fig. 1a, DLS study showed that SP/GOx NPs was mono-dispersed in water with an average diameter about $255 \mathrm{~nm}$. From TEM image, we found that SP/GOx NPs was in uniform spherical structure and the diameter distribute from 200 to $350 \mathrm{~nm}$ (Fig. 1b), which consist with the DLS investigation. Furthermore, the chemical composition of SP/ GOx NPs was revealed by energy dispersive X-ray spectroscopy (EDS) mapping. As shown in Fig. 1d-h, C, O, $\mathrm{Fe}, \mathrm{S}, \mathrm{N}$ elements were dispersed homogeneously in $\mathrm{SP} /$ GOx NPs, demonstrating they are from AP5, FeE and GOx.

\section{Chemodynamic performance of SP/GOx NPs}

As we all known that $\mathrm{Fe}^{2+}$ ion is a classical Fenton-like catalyst, which can catalysis $\mathrm{H}_{2} \mathrm{O}_{2}$ to generate $\cdot \mathrm{OH}$ to kill cancer cells in tumor issues and to achieve chemodynamic therapy (CDT) [68]. Here, we used 3,3,5,5'-tetramethylbenzidine (TMB) to detect the $\cdot \mathrm{OH}$ generation ability of SP/GOx NPs due to non-fluorescent TMB can be oxidized by $\cdot \mathrm{OH}$ to form fluorescent ox-TMB. As shown in Fig. 2a, in the absence of SP/GOx NPs, there was no absorbance peak of the solution of $\mathrm{H}_{2} \mathrm{O}_{2}$ \& TMB (light blue line). However, upon addition of SP/GOx NPs, the absorbance peak at $650 \mathrm{~nm}$ was observed (red line), suggesting the generation of $\bullet \mathrm{OH}$. The more SP/GOx NPs added, the faster $\cdot \mathrm{OH}$ produced (Fig. 2a, blue/green/pink line). Furthermore, electron paramagnetic resonance (EPR) spectra were performed to verify the formed $\bullet \mathrm{OH}$ with 5,5-dimethly-1-proline-N-oxide (DMPO) as the detector [69]. In the GO' PBS solution, •OH signal can be found clearly upon addition of SP/GOx NPs, as shown in Fig. 2b (blue line). For comparison, if there is no GO in the PBS solution, no $\cdot \mathrm{OH}$ was generated (Fig. $2 \mathrm{~b}$, red line). The intracellular $\cdot \mathrm{OH}$ generation in HeLa cells is further confirmed by adopting DCFH-DA as the fluorescence indicator. As shown in Fig. 2c, in PBS group, HeLa cells showed no fluorescence, while in SP NPs group, week green fluorescence was observed due to the Fenton activity by consuming the endogenous $\mathrm{H}_{2} \mathrm{O}_{2}$. However, when HeLa cells are incubated with SP/GOx NPs, strong green emission was observed. This is due to GOx can catalyst the over-expressed GO in cancer cells to form $\mathrm{H}_{2} \mathrm{O}_{2}$, which then further catalyst by $\mathrm{Fe}^{2+}$ through Fenton reaction to generate $\cdot \mathrm{OH}$ continuously. 


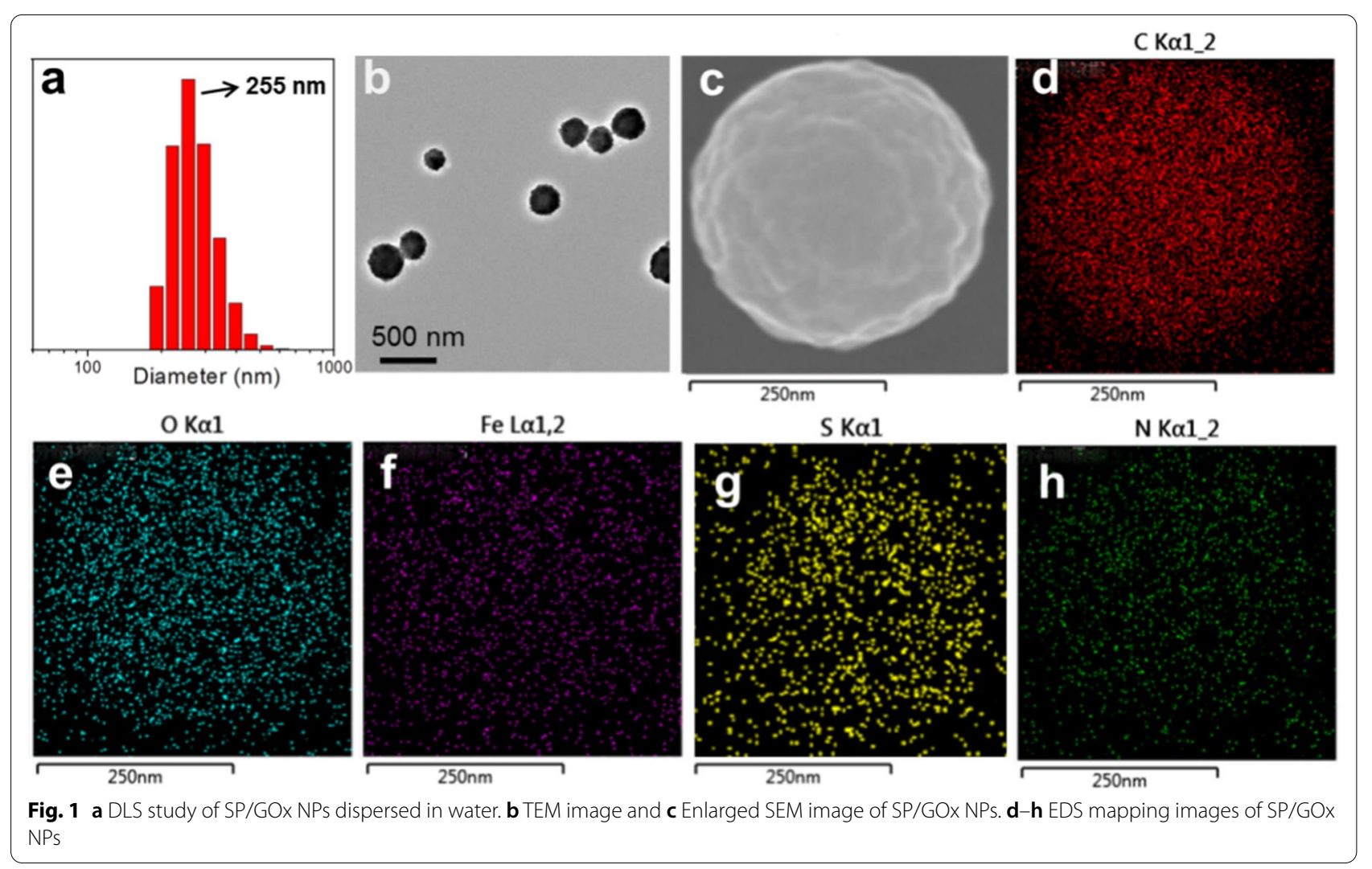

\section{Cytotoxicity of FA-Py/SP/Gox/Dox NPs toward cancer cells}

In the process of traditional tumor chemotherapy, the poor water solubility and high toxicity of drugs greatly inhibit the therapeutic effect. In this work, in order to further explore the potential application of the obtained SP/GOx NPs as a drug carrier, the drug loading and in vitro release was carried out with Doxorubicin hydrochloride (Dox) as the drug molecule. As shown in Additional file 1: Fig. S7, after adding $9.5 \mathrm{mg}$ SP/GOx NPs into the solution contain $4.5 \mathrm{mg}$ Dox and stirred $24 \mathrm{~h}$, the color of the solution became weak, indicating that Dox was loaded by SP/GOx NPs. Finally, it was calculated that approximately $3.74 \mathrm{mg}$ Dox was adsorbed and loaded in SP/GOx NPs $(394 \mu \mathrm{g} / \mathrm{mg})$. The successful preparation of SP/GOx/Dox NPs was also confirmed by UV and FT-IR spectra (Additional file 1: Fig. S13). The release behaviors of SP/GOx/Dox NPs were evaluated in PBS with $\mathrm{pH}$ 7.0, 6.0, 4.8, and 3.5, respectively (Fig. 3a). The real-time drug release profiles of Dox were monitored by UV-vis spectrophotometry. After $8 \mathrm{~h}$, the cumulative release rate reached $10.0 \%$ at $\mathrm{pH} 7.0,23.2 \%$ at $\mathrm{pH} 6.0,46.1 \%$ at $\mathrm{pH}$ 4.8 , and $76.1 \%$ at $\mathrm{pH} 3.5$, suggesting that SP/GOx/Dox NPs can be used as a sustained release carrier for cancer therapy.

More importantly, besides applied as drug carrier, SP/ GOx NPs can further modified with targeted molecules
(FA-Py) onto its surface to form FA-Py/SP/GOx/Dox NPs due to its internal host-guest interaction of pillar[5] arene framework (Additional file 1: Fig. S8 and S9), thus achieving targeted treatment of tumors. In vivo imaging of the biodistribution of the SP/Dox NPs and FA-Py/SP/ Dox NPs clearly confirmed the tumor targeting ability after FA-Py modification (Additional file 1: Fig. S12). Furthermore, confocal laser scanning microscope (CLSM) was used to investigate the cellular uptake of the resultant SP/GOx/Dox NPs and FA-Py/SP/GOx/Dox NPs by Hela cells. As shown in Additional file 1: Fig. S10, the nucleus were stained by blue fluorescent DAPI to locate the predetermined cells. When treated with FA-Py/SP/GOx/ Dox NPs, the cells exhibited more bright red fluorescence than that treated with SP/GOx/Dox NPs under the same conditions, indicating that FA-Py can target the materials to cancer cell efficiently. In addition, the merge image of NPs clearly showed that after $4 \mathrm{~h}$ of incubation, the fluorescence intensity of the cells was higher than that of those treated for $1 \mathrm{~h}$, confirming that cellular uptake of FA-Py/SP/GOx/Dox NPs was a time-dependent process. To investigate the in vitro targeted and combined chemochemodynamic cancer therapy of the resultant FA-Py/SP/ GOx/Dox NPs, HeLa cells was selected, and its viability after treating with different groups was also investigated via 3-(4,5-dimethylthiazol-2-yl)-2,5-diphenyltetrazolium 


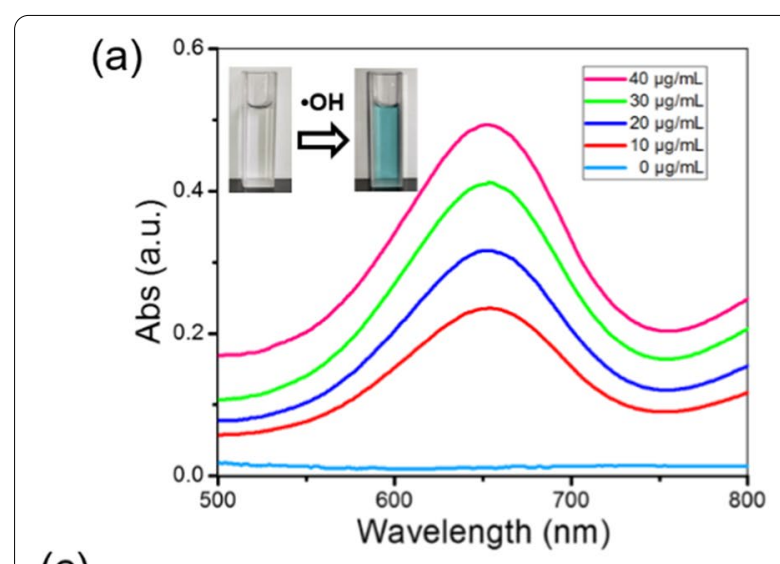

(c)
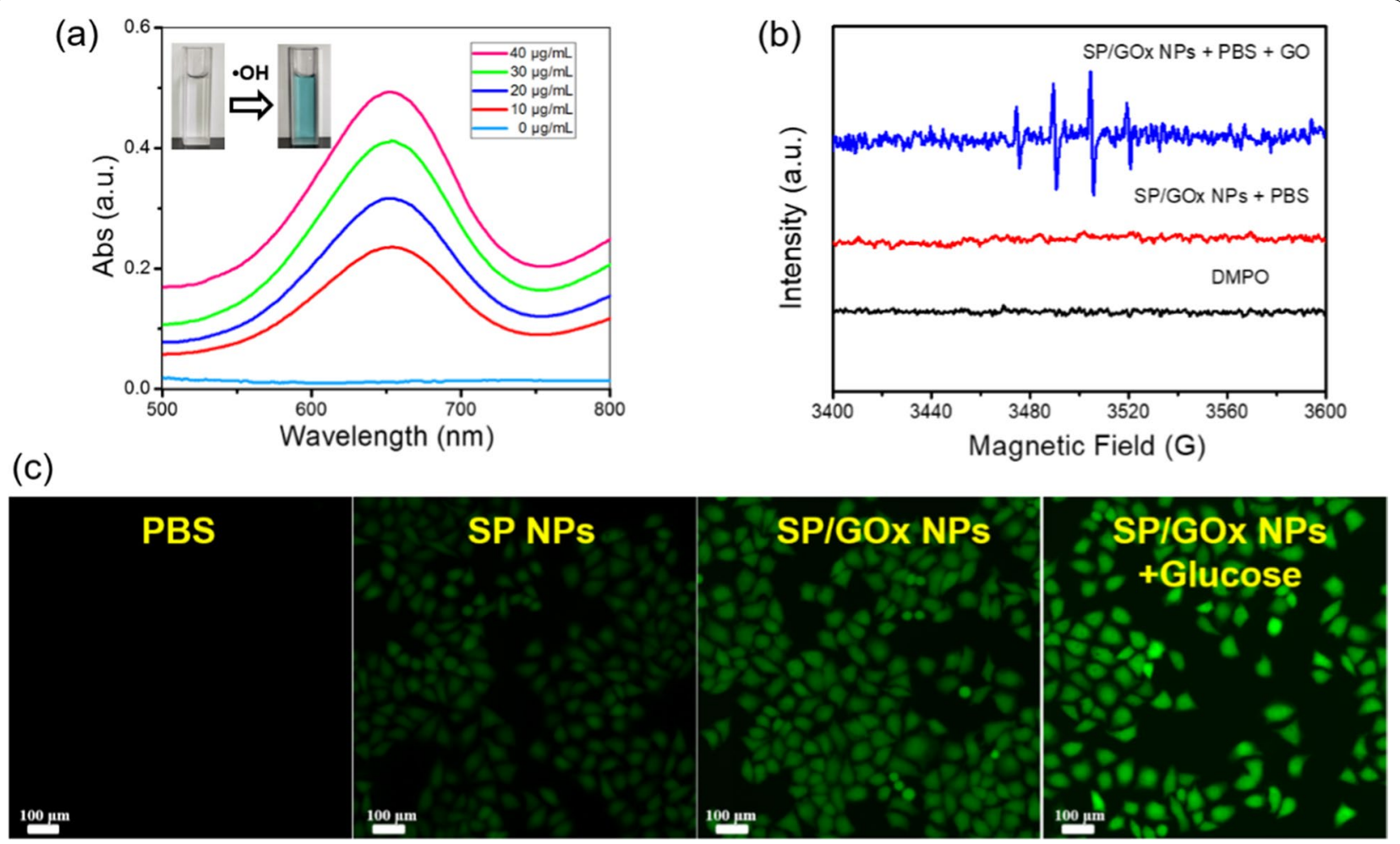

Fig. 2 a Time-dependent UV-visible spectra of the solution containing SP/GOx NPs, $\mathrm{H}_{2} \mathrm{O}_{2}$, and TMB ([TMB] $=0.25 \mathrm{mmol},\left[\mathrm{H}_{2} \mathrm{O}_{2}\right]=1.00 \mathrm{mmol}$, SP/ GOx NPs: $0.15 \mathrm{mg}$ ). $\mathbf{b}$ Electron spin resonance spectra of $\cdot \mathrm{OH}$ trapped by DMPO in SP/GOx NPs + PBS + GO (blue line), SP/GOx NPs + PBS (red line) and PBS (black line). c CLSM image of •OH generation in HeLa cells after different treatments with DCFH-DA as the probe

bromide (MTT) assays. Firstly, cells were incubated with various concentrations $(0-120 \mu \mathrm{g} / \mathrm{mL})$ of SP NPs, SP/ GOx NPs, FA-Py/SP/GOx NPs, SP/Dox NPs, FA-Py/SP/ Dox NPs, and FA-Py/SP/GOx/Dox NPs.

As shown in Fig. 3b, SP NPs exhibit high cell viability $(80 \%)$ even when the concentration is $120 \mu \mathrm{g} / \mathrm{mL}$ due to only over expressed $\mathrm{H}_{2} \mathrm{O}_{2}$ in tumor issues transformed into $\cdot \mathrm{OH}$ by the contained ferrocene units. SP/GOx NPs showed lower cell viability than SP NPs under the same conditions due to GOx can catalyst the over expressed GO in tumor cells into $\mathrm{H}_{2} \mathrm{O}_{2}$ continuously. For FA-Py/ SP/GOx NPs, FA-Py molecule can target the particles to cancer cells efficiently, so the cell viability further decreased. Importantly, after loading Dox, all the groups exhibited higher cytotoxicity, especially for the resultant FA-Py/SP/Gox/Dox NPs, the cell viability decreased to $6 \%$ when its concentration increased to $120 \mu \mathrm{g} / \mathrm{mL}$. Furthermore, to visualize the targeted and combined chemochemodynamic cancer therapy effect of FA-Py/SP/GOx/ Dox NPs, calceinacetoxymethyl (Calcein-AM) and propidium iodide (PI) staining was employed to differentiate dead (red) and live (green) cells (Fig. 3c). The cells treated with SP NPs showed almost bright green fluorescence, indicating they lived well. Nevertheless, cells incubated with FA-Py/SP/GOx/Dox NPs were all died and exhibited red fluorescence. All the above results confirmly certified the excellent therapeutic effect of FA-Py/SP/GOx/ Dox NPs.

\section{In vivo targeted chemo-chemodynamic therapy efficacy of FA-Py/SP/GOx/Dox NPs}

Inspired by the outstanding therapeutic effect of FA-Py/SP/GOx/Dox NPs in vitro, the in vivo antitumor efficacy on mouse was also performed. Firstly, the mice were divided into 7 groups (Control, SP NPs, SP/GOx NPs, FA-Py/SP/GOx NPs, SP/Dox NPs, FA-SP/Dox $\mathrm{NPs}$, and FA-Py/SP/GOx/Dox NPs) randomly when the size of tumors reached $\sim 100 \mathrm{~mm}^{3}$. As shown in Fig. 4a, there was no significant change in body weight in all groups during the treatment period, indicating that the this pillar[5]arene based nanomaterials are reasonably safe. Then the tumor volumes of mice were monitored every three days to check the cancer therapy activity of different groups. As shown in Fig. 4b, the tumor volume increased rapidly for the mice in Control group, while the SP NPs, SP/GOx NPs and FA-Py/SP/GOx NPs showed a slight tumor suppressive effect. However, The cancer therapy efficacy of SP/Dox NPs was better 


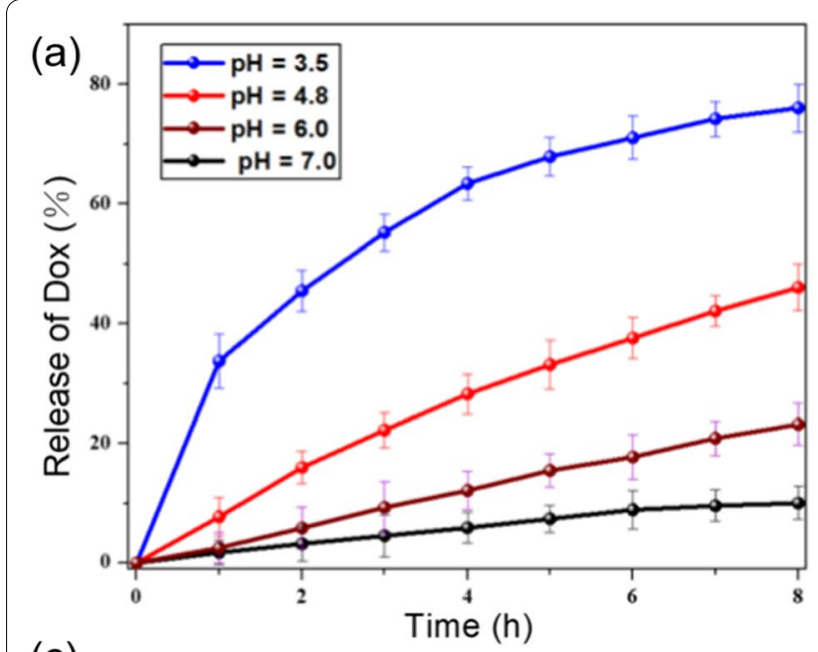

(c)
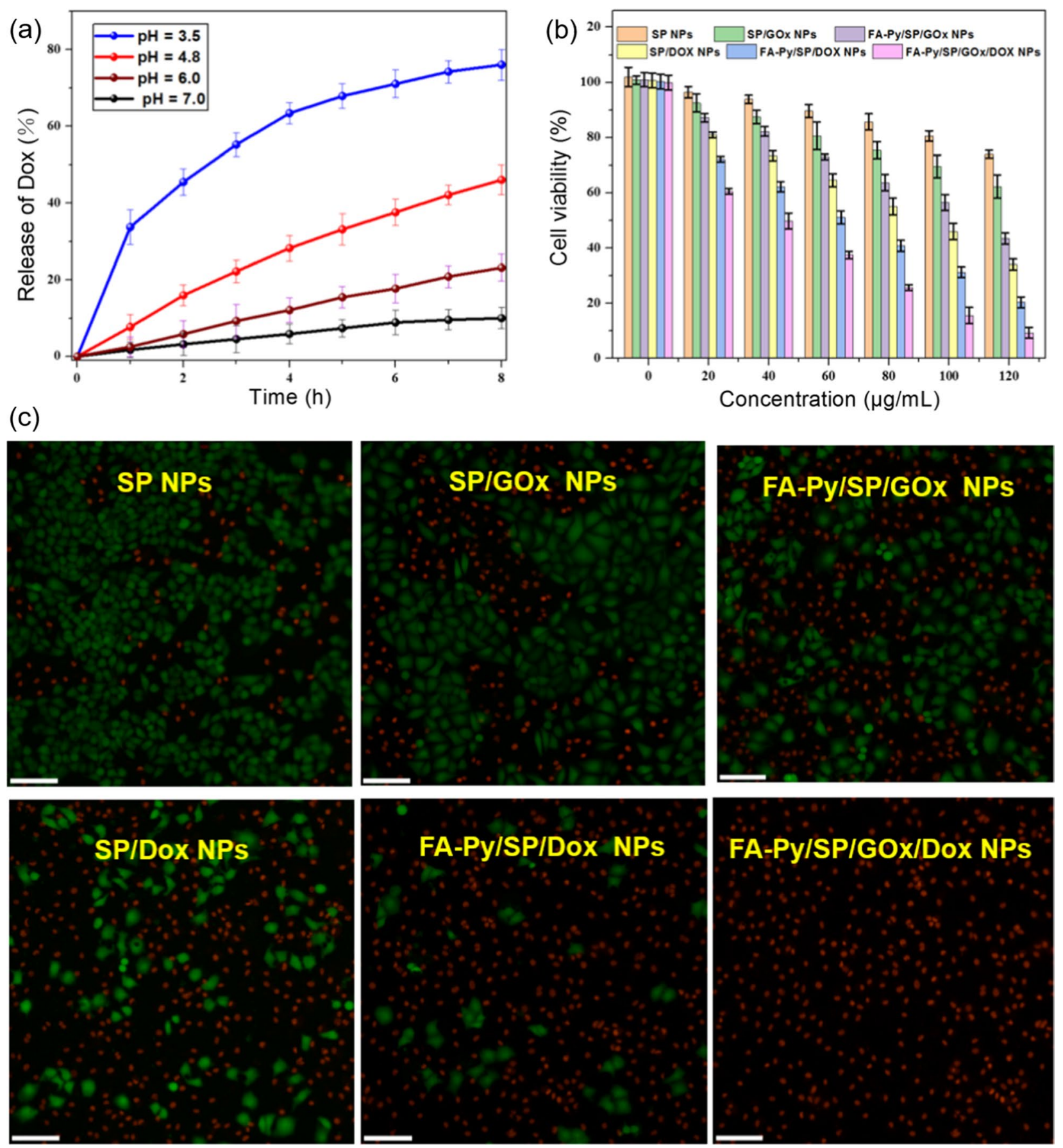

Fig. 3 a Dox release profiles from SP/GOx NPs at pH 3.5, pH 4.8, pH 6.0 and pH 7.4, respectively. $\mathbf{b}$ Cell viabilities of HeLa cells incubated with SP NPs, SP/GOx NPs, FA-Py/SP/GOx NPs, SP/Dox NPS, FA-Py/SP/Dox NPs, and FA-Py/SP/GOx/Dox NPs at different concentrations. c Fluorescence images of Calcein AM (live cells, green) and PI (dead cells, red) costained HeLa cells after different groups

than those of SP NPs but lower than the FA-Py/SP/Dox NPs, demonstrating the important role of the target molecule FA-Py. Significantly, FA-Py/SP/GOx/Dox NPs showed the best inhibitory effect to tumor, indicating the excellent therapeutic effect of the targeted and combined chemo-chemodynamic cancer therapy.

The photograph of the tumors treated with different treatments was observed in Fig. 4c. The Control group had the largest tumor, followed by SP NPs group, SP/ 

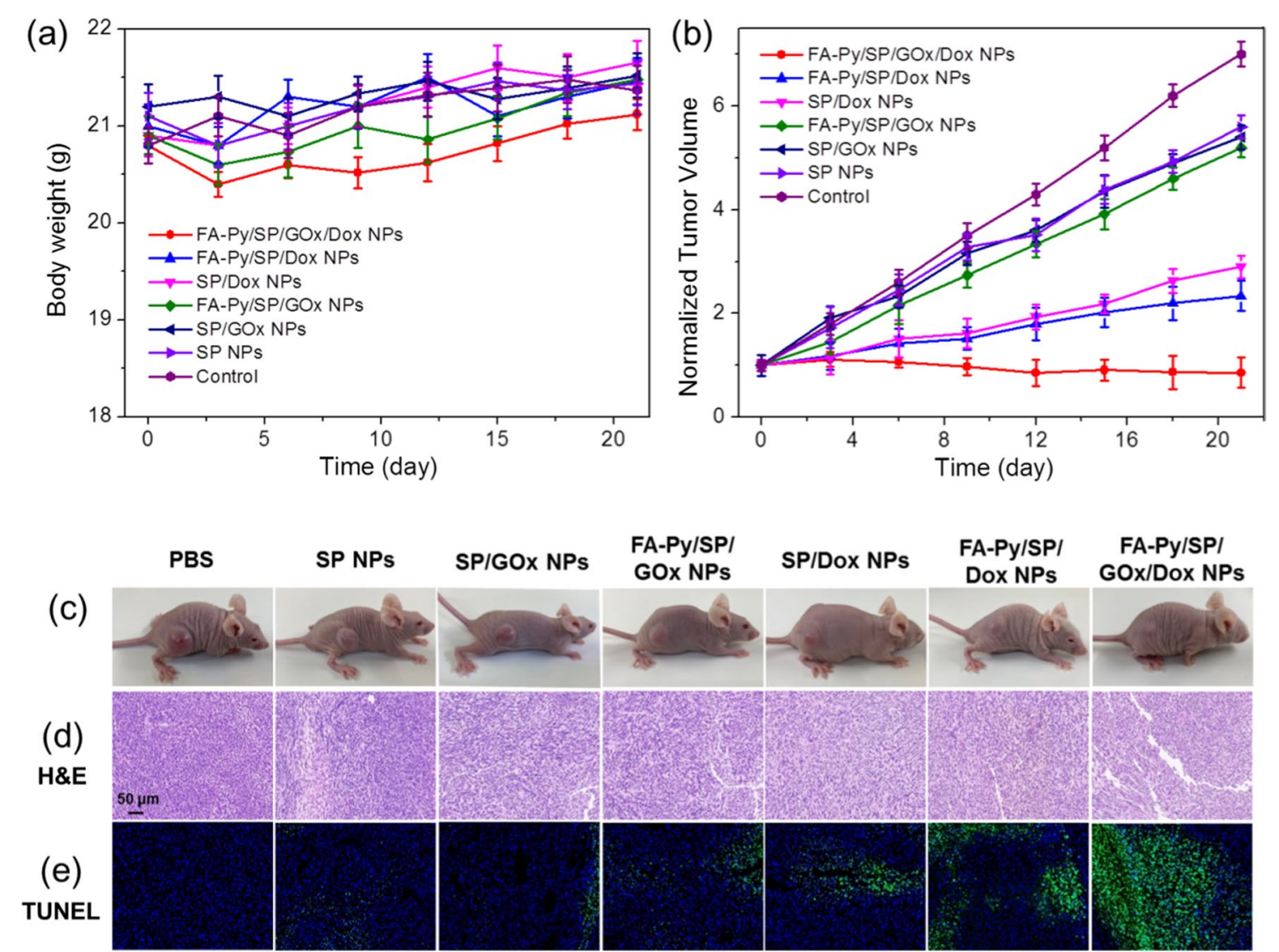

Fig. 4 a Body weight curves and $\mathbf{b}$ normalized tumor volume curves of tumor-bearing KM mice in different treatment groups. c Representative tumor photograph at 21th day. $\mathbf{d}$ H\&E and $\mathbf{e}$ TUNEL staining of tumors in corresponding groups

GOx NPs group, and FA-Py/SP/GOx NPs group. SP/Dox NPs group and FA-Py/SP/Dox NPs group were smaller. However, FA-Py/SP/GOx/Dox NPs group showed the smallest tumor, which consist with the above tumor volume results. Furthermore, from H\&E and TUNEL staining assay (Fig. 4d, e) we found that the FA-Py/SP/GOx/ Dox NPs group showed the most prominent reduction in tumor cells, and the most TUNEL-positive tumor cells. Then in order to investigate the side effects on the major organs of the mice, the H\&E staining of heart, liver, spleen, lung, and kidney were performed, which confirmed that no obvious inflammatory damage and tissue damage in all major organs (Additional file 1: Fig. S11). All the results certified that this pillar[5]arene based supramolecular materials possess excellent efficiency for cancer therapy with good biocompatibility.

\section{Conclusion}

In conclusion, pillar[5]arene-based supramolecular polymeric materials (SP/GOx NPs) was prepared from AP5 and FeE with the assistant of GOx. NMR, SEM,
TEM and EDS-Mapping were applied to characterize its morphology and chemical compositions. The obtained SP/GOx NPs can load drug molecules (Dox) and modify target molecule (FA-Py) on its surface due to it possess both the advantages of adsorption ability and rich host-guest interaction. In this case, when the resultant FA-Py/SP/GOx/Dox NPs enters blood circulation, FA-Py will target it to cancer cells efficiently, where GOx can catalyst the overexpressed GO to generate $\mathrm{H}_{2} \mathrm{O}_{2}$. Subsequently, the generated $\mathrm{H}_{2} \mathrm{O}_{2}$ in cancer cells catalyzed by ferrocene unit to form $\cdot \mathrm{OH}$, which can kill cancer cells. Furthermore, the loaded Dox molecules released under acid microenvironment, which can further achieve chemo-therapy. Both the in vitro and in vivo investigations certified that the excellent targeted and combined chemo-chemodynamic cancer therapy of Py/SP/GOx/Dox NPs.

\section{Supplementary Information}

The online version contains supplementary material available at https://doi. org/10.1186/s12951-021-01237-0. 
Additional file 1. Materials. Characterization. Synthesis of AP5. Construction of FA-Py/SP/GOx/Dox NPs. Cell experiments. Animal experiments.

\section{Acknowledgements}

This work was financially supported by the National Natural Science Foundation of China (21801139), the Natural Science Foundation of Jiangsu Province (BK20180942, BK20190917), the Natural Science Foundation of the Higher Education Institutions of Jiangsu Province (19KJB150015), the Six Talent Peak Projects in Jiangsu Province (XCL-085). We also thank Nantong University Analysis \& Testing Center for characterization.

\section{Authors' contributions}

JW and DW performed the experiments and wrote the draft manuscript. MC, $D J, J B, Y H$, and $J Z$, analyzed the data and drew the Schemes and Figures. BL polished the English. YW, and YY got the funding and designed the project. All authors read and approved the final manuscript.

\section{Funding}

National Natural Science Foundation of China, 21801139, Yong Yao, Natural Science Foundation of Jiangsu Province, BK20180942, Yong Yao, BK20190917, Yang Wang.

\section{Declarations}

\section{Ethics approval and consent to participate}

All animal experiments were approved by the Animal Ethics Committee of Nantong University.

\section{Consent for publication}

All authors in the paper agree to be published.

The preprint is on https://www.researchsquare.com/article/rs-1 109554/v1

\section{Competing interests}

The author declare that they have no competing interests.

Received: 25 November 2021 Accepted: 30 December 2021 Published online: 11 January 2022

\section{References}

1. Bray F, Ferlay J, Soerjomataram I, Siegel RL, Torre LA, Jemal A. Global cancer statistics 2018: GLOBOCAN estimates of incidence and mortality worldwide for 36 cancers in 185 countries. CA-Cancer J Clin. 2018;68(6):394-424.

2. Zhou J, Yu G, Huang F. Supramolecular chemotherapy based on hostguest molecular recognition: a novel strategy in the battle against cancer with a bright future. Chem Soc Rev. 2017;46:7021-53.

3. Zhou J, Rao L, Yu G, CookTR, Chen X, Huang F. Supramolecular cancer nanotheranostics. Chem Soc Rev. 2021;50:2839-91.

4. Wang L, Li L-L, Fan Y-S, Wang H. Host-guest supramolecular nanosystems for cancer diagnostics and therapeutics. Adv Mater. 2013;25(28):3888-98.

5. Webber MJ, Appel EA, Meijer EW, Langer R. Supramolecular biomaterials. Nat Mater. 2016;15:13-26.

6. Cheetham AG, Zhang P, Lin YA, Lock LL, Cui H. Supramolecular nanostructures formed by anticancer drug assembly. J Am Chem Soc. 2013;135(8):2907-10.

7. Yu G, Jie K, Huang F. Supramolecular amphiphiles based on host-guest molecular recognition motifs. Chem Rev. 2015;115(15):7240-303.

8. Appel EA, Barrio JD, Loh XJ, Scherman OA. Supramolecular polymeric hydrogels. Chem Soc Rev. 2012;41:6195-214.

9. Ogoshi T, Kanai S, Fujinami S, Yamagishi T-A, Nakamoto Y. para-bridged symmetrical pillar[5]arenes: their lewis acid catalyzed synthesis and hostguest property. J Am Chem Soc. 2008;130(15):5022-3.

10. Cao D, Kou Y, Liang J, Chen Z, Wang L, Meier H. A facile and efficient preparation of pillararenes and a pillarquinone. Angew Int Ed Chem. 2009;48(51):9721-3.
11. Zhang Z, Luo Y, Chen J, Dong S, Yu Y, Ma Z, et al. Formation of linear supramolecular polymers that is driven by $\mathrm{C}-\mathrm{H} \cdots \pi$ interactions in solution and in the solid state. Angew Int Ed Chem. 2011;50(6):1397-401.

12. Li P-Y, Chen Y, Chen C-H, Liu Y. Amphiphilic multi-charged cyclodextrins and vitamin $\mathrm{K}$ co-assembly as a synergistic coagulant. Chem Commun. 2019;55:11790-3.

13. Künnemann KU, Schurm L, Lange D, Seidensticker T, Tilloy S, Monflier $\mathrm{E}$, et al. Continuous hydroformylation of 1-decene in an aqueous biphasic system enabled by methylated cyclodextrins. Green Chem. 2020;22:3809-19.

14. Bolton SG, Pluth MD. Modified cyclodextrins solubilize elemental sulfur in water and enable biological sulfane sulfur delivery. Chem Sci. 2020;11:11777-84.

15. Lai W-F, Rogach AL, Wong W-T. Chemistry and engineering of cyclodextrins for molecular imaging. Chem Soc Rev. 2017;46:6379-419.

16. Jiang T, Qu G, Wang J, Ma X, Tian H. Cucurbiturils brighten Au nanoclusters in water. Chem Sci. 2020;11:3531-7.

17. Assaf K, Nau WM. Cucurbiturils: from synthesis to high-affinity binding and catalysis. Chem Soc Rev. 2015;44:394-418.

18. Ge Z, Hu J, Huang F, Liu S. Responsive supramolecular gels constructed by crown ether based molecular recognition. Angew Chem Int Ed. 2009;48(10):1798-802.

19. Curcio M, Nicoli F, Paltrinieri E, Fois E, Tabacchi G, Cavallo L, et al. Chemically induced mismatch of rings and stations in [3]rotaxanes. J Am Chem Soc. 2021;143(21):8046-55.

20. Fielden SDP, Leigh DA, McTernan CT, Pérez-Saavedra B, Vitorica-Yrezabal IJ. Spontaneous assembly of rotaxanes from a primary amine, crown ether and electrophile. J Am Chem Soc. 2018;140(19):6049-52.

21. Zhong Y-H, Lei Y, Huang J-F, Xiao L-M, Chen X-L, Luo T, et al. Design of an alkaline pyridyl acceptor-based calix[4]arene dye and synthesis of stable calixarene- $\mathrm{TiO}_{2}$ porous hybrid materials for efficient photocatalysis. J Mater Chem A. 2020;8:8883-91.

22. Zheng Z, Geng W-C, Gao J, Wang Y-Y, Sun H, Guo D-S. Ultrasensitive and specific fluorescence detection of a cancer biomarker via nanomolar binding to a guanidinium-modified calixarene. Chem Sci. 2018;9:2087-91.

23. Li L, Tuo W, Zhu Q, Sepehrpour H, Yao Y, Yan C, et al. Resorcinarene induced assembly of carotene and lutein into hierarchical superstructures. J Am Chem Soc. 2020;142(49):20583-7.

24. Sun C-L, Peng H-Q, Niu L-Y, Chen Y-Z, Wu L-Z, Tung C-H, et al. Artificial light-harvesting supramolecular polymeric nanoparticles formed by pillar[5]arene-based host-guest interaction. Chem Commun. 2018;54:1117-20.

25. Hao Q, Chen Y, Huang Z, Xu J-F, Sun Z, Zhang X. Supramolecular chemotherapy: carboxylated pillar[6]arene for decreasing cytotoxicity of oxaliplatin to normal cells and improving its anticancer bioactivity against colorectal cancer. ACS Appl Mater Interfaces. 2018;10(6):5365-72.

26. Ogoshi T, Furuta T, Hamada Y, Kakuta T, Yamagishi T. Solid-state selfinclusion complexation behaviour of a pillar[5]arene-based host-guest conjugate. Mater Chem Front. 2018;2:597-602.

27. Guo S, Song Y, He Y, Hu X-Y, Wang L. Highly efficient artificial light-harvesting systems constructed in aqueous solution based on supramolecular self-assembly. Angew Chem Int Ed. 2018;57(12):3163-7.

28. Wanderlind EH, Liz DG, Gerola AP, Affeldt RF, Nascimento V, Bretanha LC, et al. Imidazole-functionalized pillar[5]arenes: highly reactive and selective supramolecular artificial enzymes. ACS Catal. 2018;8(4):3343-7.

29. Yang W, Samanta K, Wan X, Thikekar TU, Chao Y, Li S, et al. Tiara[5]arenes: synthesis, solid-state conformational studies, host-guest properties, and application as nonporous adaptive crystals. Angew Chem Int Ed. 2020;59(10):3994-9.

30. Xu X, Jerca FA, Hecke KV, Jerca WV, Hoogenboom R. High compression strength single network hydrogels with pillar[5]arene junction points. Mater Horiz. 2020;7:566-73.

31. Jie K, Zhou Y, Sun Q, Li B, Zhao R, Jiang D, et al. Mechanochemical synthesis of pillar[5]quinone derived multi-microporous organic polymers for radioactive organic iodide capture and storage. Nat Commun. 2020;11:1086.

32. Wu M-X, Gao J, Wang F, Yang J, Song N, Jin X, et al. Multistimuli responsive core-shell nanoplatform constructed from $\mathrm{Fe}_{3} \mathrm{O}_{4} @ \mathrm{MOF}$ equipped with pillar[6]arene nanovalves. Small. 2018;14(17):1704440. 
33. Yu G, Yang J, Fu X, Wang Z, Shao L, Mao Z, et al. A supramolecular hybrid material constructed from graphene oxide and a pillar[6]arene-based host-guest complex as an ultrasound and photoacoustic signal nanoamplifier. Mater Horiz. 2018;5:429-35.

34. Li E, Jie K, Zhou Y, Zhao R, Huang F. Post-synthetic modification of nonporous adaptive crystals of pillar[4]arene[1]quinone by capturing vaporized amines. J Am Chem Soc. 2018;140(44):15070-9.

35. Muhammed MAH, Cruz LK, Emwas A-H, El-Zohry AM, Moosa B, Mohammed OF, Khashab NM. Pillar[5]arene-stabilized silver nanoclusters: extraordinary stability and luminescence enhancement induced by host-guest interactions. Angew Chem Int Ed. 2019;58(44):15665-70.

36. Li H, Yang Y, Xu F, Liang T, Wen H, Tian W. Pillararene-based supramolecular polymers. Chem Commun. 2019;55:271-85.

37. Gao L, Li M, Ehrmann S, Tu Z, Haag R. Positively charged nanoaggregates based on zwitterionic pillar[5]arene that combat planktonic bacteria and disrupt biofilms. Angew Chem Int Ed. 2019;58(11):3645-9.

38. Jie K, Liu M, Zhou Y, Little MA, Pulido A, Chong SY, et al. Near-ideal xylene selectivity in adaptive molecular pillar[n]arene crystals. J Am Chem Soc. 2018;140(22):6921-30.

39. Shen $Y$, Song W, Barden DR, Ren $T$, Lang C, Feroz H, et al. Achieving high permeability and enhanced selectivity for Angstrom-scale separations using artificial water channel membranes. Nat Commun. 2018;9:2294.

40. Wu J, Tian J, Rui L, Zhang W. Enhancing the efficacy of photodynamic therapy (PDT) via water-soluble pillar[5]arene-based supramolecular complexes. Chem Commun. 2018;54:7629-32.

41. Zhang H, Liu Z, Zhao Y. Pillararene-based self-assembled amphiphiles. Chem Soc Rev. 2018;47:5491-528.

42. Kakuta T, Yamagishi T-A, Ogoshi T. Stimuli-responsive supramolecular assemblies constructed from pillar[n]arenes. Acc Chem Res. 2018;51(7):1656-66.

43. Lee E, Ju H, Park I-H, Jung JH, Ikeda M, Kuwahara S, et al. Pseudo[1] catenane-type pillar[5]thiacrown whose planar chiral inversion is triggered by metal cation and controlled by anion. J Am Chem Soc. 2018;140(30):9669-77.

44. Yao Y, Wei X, Cai Y, Kong X, Chen J, Wu J, et al. Hybrid supramolecular materials constructed from pillar[5]arene based host-guest interactions with photo and redox tunable properties. J Colloid Interf Sci. 2018;525(1):48-53.

45. Yang K, Yang K, Chao S, Wen J, Pei Y, Pei Z. A supramolecular hybrid material constructed from pillar[6]arene-based host-guest complexation and ZIF-8 for targeted drug delivery. Chem Commun. 2018;54:9817-20.

46. Zuo M, Qian W, Xu Z, Shao W, Hu X-Y, Zhang D, et al. Multiresponsive supramolecular theranostic nanoplatform based on pillar[5]arene and diphenylboronic acid derivatives for integrated glucose sensing and insulin delivery. Small. 2018;14(38):1801942.

47. Guo F, Xiao P, Yan B, Hahn M, Kong Y, Zhang W, et al. One-pot synthesis of hydrazide-pillar[5]arene functionalized reduced graphene oxide for supercapacitor electrode. Chem Eng J. 2020;391(1):123511.

48. Li Y, Segawa Y, Yagi A, Itami K. A nonalternant aromatic belt: methylenebridged [6]cycloparaphenylene synthesized from pillar[6]arene. J Am Chem Soc. 2020;142(29):12850-6.

49. Tuo W, Sun Y, Lu S, Li X, Sun Y, Stang PJ. Pillar[5]arene-containing metallacycles and host-guest interaction caused aggregation-induced emission enhancement platforms. J Am Chem Soc. 2020;142(40):16930-4.

50. Kiruthika J, Srividhya S, Arunachalam M. Anion-responsive pseudo[3] rotaxane from a difunctionalized pillar[4]arene[1]quinone and a bisimidazolium cation. Org Lett. 2020;22(20):7831-6.

51. Lee E, Park I-H, Ju H, Kim S, Jung JH, Habata Y, et al. Formation of a Pillar[5]arene-based two-dimensional poly-pseudo-rotaxane: threading and crosslinking by the same guest molecules. Angew Chem Int Ed. 2019;58(33):11296-300

52. Chen J-F, Liu X, Ma J-F, Han B-B, Ding J-D, Lin Q, et al. A pillar[5]arenebased multiple-stimuli responsive metal-organic gel was constructed for facile removal of mercury ions. Soft Matter. 2017;13:5214-8.

53. Hu W-B, Cai H-R, Hu W-J, Zhao X-L, Liu Y, Li J-S, et al. Pillar[5]arene-Py-Cu Gel, the First Pillar[5]arene-Based Metallo(organo)gel, and Adsorption of Sudan III by Its Gel-Precipitate. Eur J Inorg Chem. 2017;29:3551-4.

54. Liu Y, Shangguan L, Wang H, Xia D, Shi B. A supramolecular polymer network gel with stimuli-responsiveness constructed by orthogonal metal ion coordination and pillar[5]arene-based host-guest recognition. Polym Chem. 2017;8:3783-7.
55. Shurpik DN, Aleksandrova YI, Rodionov AA, Razina EA, Gafurov MR, Vakhitov IR, et al. Metallo-supramolecular coordination polymers based on amidopyridine derivatives of pillar[5]arene and $\mathrm{Cu}(\mathrm{II})$ and $\mathrm{Pd}(\mathrm{II})$ Cations: synthesis and recognition of nitroaromatic compounds. Langmuir. 2021;37(9):2942-53.

56. Li Y-F, Li Z, Lin Q, Yang Y-W. Functional supramolecular gels based on pillar[n]arene macrocycles. Nanoscale. 2020;12:2180-200.

57. Guo S, Liang T, Song Y, Cheng M, Hu X-Y, Zhu J-J, et al. Supramolecular polymersomes constructed from water-soluble pillar[5]arene and cationic poly(glutamamide)s and their applications in targeted anticancer drug delivery. Polym Chem. 2017;8:5718-25.

58. Zhang R, Yan X, Guo H, Hu L, Yan C, Wang Y, Yao Y. Supramolecular polymer networks based on pillar[5]arene: synthesis, characterization and application in the Fenton reaction. Chem Commun. 2020;56:948-51.

59. Zhang H, Nguyen $K$, Ma X, Yan H, Guo J, Zhu L, Zhao Y. Host-guest complexation driven dynamic supramolecular self-assembly. Org Biomol Chem. 2013;11:2070-4.

60. Xia B, Zheng B, Han C, Dong S, Zhang M, Hu B, et al. A novel pH-responsive supramolecular polymer constructed by pillar[5]arene-based hostguest interactions. Polym Chem. 2013;4:2019-24.

61. Wang X, Han K, Li J, Jia X, Li C. Pillar[5]arene-neutral guest recognition based supramolecular alternating copolymer containing [c2]daisy chain and bis-pillar[5]arene units. Polym Chem. 2013;4:3998-4003.

62. Wang S, Wang Y, Chen Z, Lin Y, Weng L, Han K, et al. The marriage of endo-cavity and exo-wall complexation provides a facile strategy for supramolecular polymerization. Chem Commun. 2015;51:3434-7.

63. Zhou Y, Jie K, Shi B, Yao Y. A Y-ray and dual redox-responsive supramolecular polymer constructed by a selenium containing pillar[5]arene dimer and a neutral guest. Chem Commun. 2015;51:11112-4.

64. Wu X, Yu Y, Gao L, Hu X-Y, Wang L. Stimuli-responsive supramolecular gel constructed by pillar[5]arene-based pseudo[2]rotaxanes via orthogonal metal-ligand coordination and hydrogen bonding interaction. Org Chem Front. 2016;3:966-70.

65. Ni M, Zhang N, Xia W, Wu X, Yao C, Liu X, et al. Dramatically promoted swelling of a hydrogel by pillar[6]arene-ferrocene complexation with multistimuli responsiveness. J Am Chem Soc. 2016;138(20):6643-9.

66. Cai Y, Yan X, Wang S, Zhu Z, Cen M, Ou C, et al. Pillar[5]arene-Based 3D Hybrid Supramolecular Polymer for Green Catalysis in Water. Inorg Chem. 2021;60(5):2883-7.

67. Xiao T, Wang L. Recent advances of functional gels controlled by pillar[n]arene-based host-guest interactions. Tetrahedron Lett. 2018;59(13):1172-82.

68. Li F, Zang M, Hou J, Luo Q, Yu S, Sun H, et al. Cascade catalytic nanoplatform constructed by laterally-functionalized pillar[5]arenes for antibacterial chemodynamic therapy. J Mater Chem B. 2021;9:5069-75.

69. Luo B, Chen L, Hong Z, You X, Huang F-P, Bian H-D, et al. A simple and feasible atom-precise biotinylated $\mathrm{Cu}(\mathrm{I})$ complex for tumor-targeted chemodynamic therapy. Chem Commun. 2021;57:6046-9.

\section{Publisher's Note}

Springer Nature remains neutral with regard to jurisdictional claims in published maps and institutional affiliations.

Ready to submit your research? Choose BMC and benefit from:

- fast, convenient online submission

- thorough peer review by experienced researchers in your field

- rapid publication on acceptance

- support for research data, including large and complex data types

- gold Open Access which fosters wider collaboration and increased citations

- maximum visibility for your research: over $100 \mathrm{M}$ website views per year

At BMC, research is always in progress.

Learn more biomedcentral.com/submissions 